\title{
The EPA's human exposure research program for assessing cumulative risk in communities
}

\author{
VALERIE G. ZARTARIAN AND BRADLEY D. SCHULTZ \\ U.S. Environmental Protection Agency, Office of Research and Development, National Exposure Research Laboratory, Research Triangle Park, \\ North Carolina, USA
}

Communities are faced with challenges in identifying and prioritizing environmental issues, taking actions to reduce their exposures, and determining their effectiveness for reducing human health risks. Additional challenges include determining what scientific tools are available and most relevant, and understanding how to use those tools; given these barriers, community groups tend to rely more on risk perception than science. The U.S. Environmental Protection Agency's Office of Research and Development, National Exposure Research Laboratory (NERL) and collaborators are developing and applying tools (models, data, methods) for enhancing cumulative risk assessments. The NERL's "Cumulative Communities Research Program" focuses on key science questions: (1) How to systematically identify and prioritize key chemical stressors within a given community?; (2) How to develop estimates of exposure to multiple stressors for individuals in epidemiologic studies?; and (3) What tools can be used to assess community-level distributions of exposures for the development and evaluation of the effectiveness of risk reduction strategies? This paper provides community partners and scientific researchers with an understanding of the NERL research program and other efforts to address cumulative community risks; and key research needs and opportunities. Some initial findings include the following: (1) Many useful tools exist for components of risk assessment, but need to be developed collaboratively with end users and made more comprehensive and user-friendly for practical application; (2) Tools for quantifying cumulative risks and impact of community risk reduction activities are also needed; (3) More data are needed to assess community- and individual-level exposures, and to link exposure-related information with health effects; and (4) Additional research is needed to incorporate risk-modifying factors ("non-chemical stressors") into cumulative risk assessments. The products of this research program will advance the science for cumulative risk assessments and empower communities with information so that they can make informed, cost-effective decisions to improve public health.

Journal of Exposure Science and Environmental Epidemiology (2010) 20, 351-358; doi:10.1038/jes.2009.20; published online 15 April 2009

Keywords: EPA, cumulative, exposure, communities, risk, community-based

\section{Background}

People want to know what their health risks are from the multiple stressors they are exposed to every day, including environmental pollutants, and how to prevent or mitigate those risks. Communities and individuals within them are faced with the challenges of identifying and prioritizing environmental issues, determining what tools are available to assist them, understanding how to use those tools to make more informed science-based decisions, and implementing risk reduction actions. Tools as defined here include information, strategies, exposure models, databases, sampling/analytical methods, and geographic information system (GIS) maps. Addressing these needs and protecting the health of Americans from environmental pollutants is a key goal of the U.S. Environmental Protection Agency (EPA)

1. Address all correspondence to: Brad Schultz, U.S. Environmental Protection Agency, Office of Research and Development, E205-02 Research Triangle Park, NC, USA.

Fax: +919541 9444. E-mail: schultz.brad@epa.gov

Received 23 October 2008; revised 30 January 2009; accepted 2 February 2009; published online 15 April 2009 policies and programs. As indicated in the EPA's Report on the Environment (USEPA, 2008a), the Agency has taken a number of actions to fulfill this goal, including establishing the standards for pollutants in the environment, requiring sources to limit their pollution, and educating members of the public about actions they can take to protect their health. The EPA has also responded to recommendations from the National Academy of Sciences, the National Academy of Public Administration, the EPA's Science Advisory Board, and other peer reviews and requests from the EPA regions and local communities to develop guidance documents and other tools for supporting community-based cumulative risk assessments (NAPA, 2008; NAS, 2008, http://dels.nas.edu/ dels/rpt_briefs/IRA_brief_final.pdf). The EPA long-term strategic planning documents (USEPA, 2006a, b) articulate specific plans and programs for measurement-derived databases, methods, and models to better understand how people are exposed to multiple pollutants for enhanced cumulative risk assessments, and to conduct community-based risk assessments. The Agency has developed a number of guidance documents in these areas (USEPA, 2003, 2007a). In addition, research efforts and applications have been conducted by other organizations, including the Centers for 
Disease Control and Prevention (CDC), academia, and the States in many communities over the years.

Various definitions of "cumulative," "community," and other terms have been used in the literature. In this paper, cumulative exposure refers to contact between multiple stressors and a community or individuals within a community over time (aggregate exposure refers to single stressor contacts over time combined over media and exposure pathways). A community is defined here as a group of individuals in the same geographical area and/or with the same demographic attributes considered to be key factors in assessing human exposure. Stressors are referred to here as toxic agents (chemical, biological, or physical) or other factors that have potential for adversely affecting human health. Risk-modifying factors (sometimes called "nonchemical stressors") are stressors that can include sociodemographic factors (e.g., age, gender, ethnicity, income, education, occupation) and related factors (e.g., disparity in health status, psychosocial stress, exposure to violence, housing characteristics, exposure to noise pollution, inadequate access to health care, behavior and lifestyle). Cumulative risk assessment incorporates areas outside the traditional "environmental" areas (Menzie et al., 2007; deFur et al., 2007; Callahan and Sexton, 2007; USEPA, 2003, 2007a). For example, studies have shown that psychosocial stress, inadequate health-care access and utilization, exposure to noise pollution, and exposure to violence (which may in turn be a function of income, education level, and minority status) can modify the effect of chemical exposures on human health (Lemay and Piotrowski, 2002; Hood, 2005; Sapolsky, 2005; deFur et al., 2007). Communities or sub-populations with higher levels of these factors are more vulnerable to the health effects caused by environmental exposures (USEPA, 2003; Davis et al., 2005; Menzie et al., 2007; Callahan and Sexton, 2007). Identifying these vulnerable populations is key in developing risk mitigation strategies tailored to a specific community. The EPA Office of Research and Development (ORD)'s National Center for Environmental Research (NCER), which supports the Agency's extramural research in exposure, effects, risk assessment, and risk management, is issuing a request for assistance with one research area focusing on the role of these modifying factors in cumulative risk.

The Community-Based Technical Support Forum is a large workgroup within the EPA open to groups and individuals in the Agency working on technical issues to support community-based risk assessments. The Forum meets regularly and includes the EPA's Community Action for a Renewed Environment (CARE) program (www.epa. gov/care), established to address community needs for mitigating environmental risks across media (USEPA, 2005). CARE is a competitive grant and technical assistance program that was initiated in 2005 to help communities address the risks from multiple sources of toxic pollution in their environment. Through CARE, the EPA Regional Offices and Program offices (e.g., Office of Air Quality Planning and Standards, Office of Radiation and Indoor Air, Office of Water, Office of Prevention, Pesticides and Toxic Substances, Office of Environmental Justice, Office of Solid Waste, Office of Children's Health Protection and Environmental Education) collaborate, and various local organizations, including non-profits, businesses, schools and governments, create partnerships that implement local solutions to reduce cumulative human health risks. As outlined in the CARE Roadmap (USEPA, 2008b), CARE communities follow 10 steps: (1) Build a partnership; (2) Identify community concerns; (3) Identify community vulnerabilities; (4) Identify community assets; (5) Identify concerns for immediate action; (6) Organize available information; (7) Rank risks; (8) Identify potential solutions; (9) Set priorities and take action; and (10) Evaluate results and revisit priorities. The US Centers for Disease Control and Prevention (CDC) funded the development of a similar protocol for their community projects called PACE EH (Protocol for Assessing Excellence in Environmental Health) that leads the user through a series of steps to engage the public, collect necessary and relevant information pertaining to community environmental health concerns, rank issues, and set local priorities for action (NACCHO, 2000; http:// www.naccho.org/topics/environmental/CEHA/documents. $\mathrm{cfm})$. The CARE Level I projects focus on steps 1-8; CARE Level II projects focus primarily on steps 9 and 10, and promote a self-sustaining process. Many Level I communities need help particularly with steps 2,6 , and 7 of the CARE Roadmap, and many Level II communities need help with quantifying the impact of their actions (step 10).

The ORD has been working closely with the CARE program to identify and address key needs to support cumulative community risk reduction efforts. A major goal of ORD's research is to develop, evaluate, and apply tools for estimating exposures to multiple stressors that will lead to cumulative risks (USEPA, 2006b). Research is specifically focused toward understanding individual and/or communitylevel exposures and risks.

The focus of this paper is the research program being conducted by the ORD's National Exposure Research Laboratory (NERL) and collaborators to address some of the many ongoing research needs related to assessing cumulative risk in communities. The overall objective of the NERL's cumulative research program is to develop, evaluate, and apply exposure models and related tools for conducting cumulative exposure assessments to support future real-world exposure, health, risk reduction, or "accountability" (i.e., impact assessment) programs. The anticipated broad range of applications (for different populations, spatial scales, and temporal scales) will require both screening level and higher tier tools that can be used to characterize, prioritize, and predict cumulative exposures and 
health risks. The EPA CARE program and the EPA Community-Based Technical Support Forum have provided important resources and feedback that have helped to shape the ORD NERL's Cumulative Communities Research Program (hereafter CCRP), which is the focus of this paper. The NERL collaborates with other laboratories and centers in ORD (e.g., the National Center for Environmental Assessment, the National Center for Environmental Research, the National Health and Environmental Effects Research Laboratory, the National Center for Computational Toxicology, the National Risk Management Research Laboratory) that are involved in cumulative risk and/or community-based risk research, as well as the partners in the CARE program. Despite the EPA's significant research commitment to this topic, the research needs and opportunities are enormous, and unlikely to be met solely by the EPA in the near future; abundant research opportunities exist for understanding cumulative risks in the real world to stressors from toxic substances and other factors.

The CCRP focuses on exposure tools for advancing the science and understanding of cumulative risk to communities and individuals. It is being driven by community needs, and involves many in-house researchers as well as partners inside and outside of the EPA. The objective of this paper is to provide community partners and scientific researchers with an understanding of (1) the conceptual framework and implementation of the CCRP and other efforts to address cumulative community risks, and (2) key data and research needs and opportunities in these areas. The following sections describe the approach for the development of the CCRP, the review and application of existing tools, the development of new tools, and future research plans and needs.

\section{Approach}

The development of this research program included defining the goals, identifying stakeholders and research needs, formulating key science questions, developing tools to address the science questions, applying the tools to case studies, and communicating results to partners and stakeholders.

\section{Specifying Goals and Objectives}

The overall goal of the EPA CCRP is to develop, apply, and provide exposure tools for advancing the science and understanding of cumulative risk. There are three primary objectives: (1) to develop tools for estimating human exposures to multiple chemical stressors that are most likely to impact cumulative risks; (2) to apply, evaluate, and demonstrate these exposure tools through selected community case studies; and (3) to communicate research findings and provide the tools to stakeholders.

The focus of the CCRP is primarily chemical stressors and the exposure component of the source-to-outcomes human health continuum (source $\rightarrow$ concentration $\rightarrow$ exposure $\rightarrow$ dose $\rightarrow$ risk $\rightarrow$ outcomes); however, NERL is collaborating with other groups in the ORD and the EPA focusing on other stressors (biological and physical) and source-tooutcomes components (e.g., estimating dose and risk from human exposure information). We also recognize that relevant information on sociodemographic factors (e.g., age, gender, ethnicity, income, education, occupation) and related risk-modifying factors (e.g., disparity in health status, psychosocial stress, exposure to violence, housing characteristics, exposure to noise pollution, inadequate access to health care, behavior and lifestyle) is needed for a more holistic understanding of cumulative human health risks. Thus, the CCRP researchers look to CARE partners and other collaborators (e.g., the ORD National Center for Environmental Research, the EPA Office of Environmental Justice, academia) to help develop papers discussing promising practices for incorporating these stressors and community vulnerabilities along with other environmental risks into a cumulative characterization for communities.

To estimate cumulative exposures and risks, there needs to be a common metric for this summation, even if not completely quantitative. In addition, there could be a myriad of stressors causing an adverse health effect. To effectively address cumulative risks, we are focusing research primarily on stressors related to high impact human health effects. In the case of mortality, the CDC has attempted to determine major causes of death (Mokdad et al., 2004, 2005). The CCRP is following some of the same principles in focusing on major exposures deemed most likely to impact human health outcomes, and to be common to many communities. The list of toxic substance stressors to be considered for quantitative exposure and risk characterizations focuses on those for which the EPA has at least partial responsibility for regulating, providing outreach, or conducting other actions, and is based, in part, on various comparative risk projects conducted by the EPA and the States (USEPA, 1987, 1990, 1993). Some of these toxic substances with high human health impacts across the population include ozone and fine particulate matter $\left(\mathrm{PM}_{2.5}\right)$ in the ambient air, lead from multiple sources, environmental tobacco smoke, and radon. Risk characterizations for other issues identified as important to communities are also being considered by the CCRP and CARE partners.

\section{Identifying Potential Stakeholders and Research Needs} A critical step in developing this CCRP was to identify key research needs through a review of the current communitybased and cumulative risk assessment science, tools, and programs (Barzyk et al., 2009; Medina-Vera et al., 2009); through a survey of CARE project officers (Barzyk et al., 2007); and through an ongoing dialogue with partners and stakeholders. The review of existing EPA tools included an extensive internet search and cross-check with the CARE Resources Guide (USEPA, 2008c). Barzyk et al. (2009) 
describes a review of existing modeling and database-related tools for assessing exposures and risks in communities. MedinaVera et al. (2009) reviews lower-cost measurement methods for assessing contaminant levels in communities.

To better understand stakeholder needs, the NERL exposure scientists and managers met with various groups within the Agency to present proposed project plans, including other ORD laboratories and centers focusing on different components of the human health source-to-outcomes continuum; the CARE Program; the Community-Based Technical Support Forum; and various EPA Program and Regional Offices. We incorporated feedback from those within the EPA engaged in community risk research, tools development, and applications to understand what tools have worked, what the critical needs are, and which needs the CCRP can address.

In addition to internal meetings and presentations, ORD/ NERL presented the CCRP at the joint 2007 conference of the International Society of Exposure Analysis and International Society of Environmental Epidemiology (Zartarian et al., 2007) and incorporated additional input from others in the scientific community. We also participated in the EPA Workshop on Research Needs for Community-Based Risk Assessment (USEPA 2007b; http://es.epa.gov/ncer/cbra/ presentations/11_18_07/proceedings.pdf). Furthermore, specific tools and case studies have been presented by Program leads to community members themselves.

\section{Formulating Key Science Questions}

To meet the Program goals and objectives, research is being conducted initially to address three science questions. Most efforts to date have focused on Science Question no. 1, "How to systematically identify and prioritize key environmental issues within a given community related to human health?" This is a major question being asked by CARE Level I projects and other EPA community programs. Science Question no. 2, "How to develop estimates of exposure at the individual level to multiple stressors for epidemiological studies?" refers to the general need for refined tools to quantify individual-level exposures to multiple stressors over time in epidemiological studies such as the National Children's Study (NCS, 2007; http://www.nationalchildrensstudy.gov/research/studydesign/ researchplan/Pages/Appendices.aspx). The basis for Science Question no. 3, "What tools can be used to assess community-level population distributions of exposure for the development and evaluation of the effectiveness of risk reduction strategies?," is the need for quantifying the impact of Agency community risk reduction programs such as CARE Level II projects.

\section{Applying Existing Tools to Case Studies}

The application of existing tools to several initial case studies was intended to serve the needs of stakeholders by providing useful community-specific information, while simultaneously providing information to inform and optimize new tools being developed by the CCRP to address critical research gaps. Developing tools through actual case studies can quickly help to identify data needs and practical issues to refine or enhance the tools. An important criterion of the tools to be developed under this Program is that they be widely applicable. Thus, the purpose of these case studies is to demonstrate the tools' utility as well as to provide important information to Regions, communities, and other stakeholders. Initial case studies include applying existing GIS mapping tools and databases to help prioritize issues for several EPA CARE Level I projects (Hammond et al., 2009; Hammond et al., 2008, http://secure.awma.org/events/iseeisea/images/Conference_Abstract_Book.pdf), and partnering with several risk reduction projects. The initial case studies demonstrate utility of the tools for answering some of the typical community questions at the national and community scales, but existing tools cannot answer many questions well and community groups generally found them difficult to use. Additional collaborative case study applications identified with our partners will be conducted to apply, refine, and demonstrate new tools developed in the CCRP.

\section{Developing New Tools to Address Key Needs}

Based on the outcome of the tools review and several case studies with available tools, new exposure-related tools are being developed to address the project goals, objectives, and key science questions. Human exposure differences are central to the differences in health risks from one community to another. Thus, many important research needs related to community-based risk assessment focus on developing the science to characterize exposure to communities and individuals in those communities. Communities are ultimately concerned about risk, or projected human health impacts, and what can be done to mitigate those risks. Therefore, multi-disciplinary approaches are required that combine various fields including emissions characterization, dispersion modeling, human time-location activity patterns, toxicology and epidemiology to characterize the risk to humans from cumulative environmental exposures. It is necessary to integrate the information in a consistent and comparable manner across different media such as air, water, or land, to provide communities with a comprehensive characterization of their environment. The next section describes the progress to date on the new tools being developed under the CCRP.

\section{Results and ongoing research}

Review and Compendium of Relevant Community Tools A detailed review and summary of various tools for community-based risk assessments was conducted and two articles were prepared as part of this CCRP. Barzyk et al. 
(2009) includes summary tables and related information for community risk assessment guidance documents, environmental issues for communities, web-based GIS tools for issue identification/prioritization, databases by media, and exposure models. This paper includes a discussion of how well tools intersect with community issues, which tools communities are currently using, general strengths and limitations of available tools, and specific research needs based on the review. The compendium of summary tables reviewed and inventoried was provided to the EPA CARE program for posting on their online CARE Resources Guide (USEPA, The Community CARE Resource Guide. Washington DC, 2008, www.epa.gov/osp/care/library/CARE_Resource Guide. pdf) and has been presented to CARE Project Officers in the EPA Regions for assisting with current CARE Level I projects. This research product will help to develop minimum requirements for CARE grantees, a significant need identified by the National Academy of Public Administration (NAPA, 2008; http://www.napawash.org/pc_management_studies/care. html), and will be useful to other community risk prioritization efforts.

Medina-Vera et al. (2009) provides an overview of inexpensive, screening-level, measurement-based sampling and analysis methods for multiple chemicals. This overview and compendium of methods will help to focus their application in selected case studies (e.g., in the National Children's Study) to supplement existing data and understand the factors that most impact cumulative risk for enhanced community risk assessments; to provide critical information to databases and exposure models for risk characterization; and to provide guidance relevant to cumulative risk assessments in a usable form for communities and other stakeholders.

\section{Development of New Community-Focused Exposure and Risk Screening Tool}

Parts of the USEPA National-Scale Air Toxics Assessment (NATA; www.epa.gov/ttn/atw/nata1999; USEPA, 2006c) (e.g., the cancer assessment) provide the type of information that can be used for understanding cumulative risks, and are an excellent building block for continued research. For most other environmental issues, however, that type of risk information is not available. Under the CCRP, NERL, in close collaboration with the CARE Program, is developing a Community-Focused Exposure and Risk Screening Tool (C-FERST) to assist communities with the challenge of environmental issue identification and prioritization for exposure and risk reduction efforts (Zartarian and Schultz, 2008;http://secure.awma.org/events/isee-isea/images/ Conference_Abstract_Book.pdf). The C-FERST is being developed as a state-of-the-science web-based tool that builds upon and/or links to existing best available EPA information and tools relevant to community risk characterizations. Initial C-FERST development has been based on the review of tools discussed above, as well as the draft 2006 EPA Community Screening of Environmental Risks: A Workbook for CARE Communities. The C-FERST will incorporate human exposure science including data, maps, model results, and local data collection methods. It will be a comprehensive "onestop shopping" tool that is easy to use and transparent, but will contain exposure-based cumulative risk characterizations based on the best available science.

This will be accomplished by estimating exposures and risks in a way that can be summed across chemical and nonchemical stressors in a comparable manner. For example, if a toxic substance is estimated to cause a certain number of health effects (e.g., lung cancer cases) in a population, C-FERST will estimate the cumulative health effects across related stressors to assist in risk prioritization. It will incorporate research being conducted by the EPA and others on chemical mixtures and the interactions and effects of risk modifying factors (e.g., "non-chemical" stressors such as noise and stress) on environmental stressors. This cumulative approach will be used to estimate exposures and risks for the different categories of issues in C-FERST: sources (e.g., airports, diesel exhaust from traffic); individual toxic substances of concern (e.g., radon, ETS, benzene); and health effects (e.g., childhood asthma, lung cancer). Where a quantitative summation is not possible across stressors, C-FERST will provide aggregate exposure or risk estimates for the user to examine collectively as part of a semiquantitative cumulative assessment until additional information exists.

The C-FERST user will be able to view a community report, for selected EPA-related environmental issues, that contains general information (e.g., fact sheets, weblinks) about the selected issue, or more specific information (e.g., technical papers, maps) about the population affected, sources, concentrations, exposures, risks, health effects, key factors, and exposure or risk reduction actions. Specific options in the prototype version that has been developed include the following: select from a list of EPA-related community environmental issues; view optional links to relevant fact sheets, data, and maps to inform issue selection decisions; view a GIS-based community profile based on available national, local, or proxy databases; link to available EPA databases and other relevant tools; link to guidance, methods, and tables on how to obtain or measure local data for key exposure or risk factors, and enter new data if desired to overlay on (but not alter) available C-FERST maps; view a characterization and/or maps of exposure-based health risks for a community, for selected issues with available data; link to technical papers and "promising practices" papers describing the risk characterizations for each issue; and explore (e.g., with tables and/or GIS maps) exposure or risk factors for selected issues and/or information (e.g., fact sheets) to assist with developing reduction actions. Science aspects for C-FERST development include the following: identifying environmental issues to include in the tool; 
identifying and compiling existing information and data on populations affected, sources, concentrations, exposures, risks, health effects, and key factors for each issue; translating existing risk information (e.g., national, other proxy) to a given community using exposure factors and available local data; and developing new exposure and risk estimates where needed.

Research to provide the scientific foundation for C-FERST is underway on assessing key exposure factors, data needs, and community exposure and risk characterization approaches for different environmental issues (e.g., benzene, radon, environmental tobacco smoke, ultraviolet radiation). Initially, the tool is being developed for EPA project officers working with community partners to more easily facilitate training for optimal use of the tool; future end users could include other federal, state, or local agencies working with community partners, and community leaders and members themselves. A prototype test version of C-FERST has been developed and presented at scientific conferences and community meetings to receive feedback from both science and usability perspectives. This feedback will be incorporated into future versions of C-FERST.

\section{Exposure Tools to Assess Individual-Level Exposures for Enhanced Epidemiological Studies}

The National Children's Study (NCS) will examine the effects of risk-modifying factors (e.g., natural and anthropogenic environment factors, biological and chemical factors, physical surroundings, social factors, behavioral influences and outcomes, genetics, cultural and family influences and differences, geographic locations) on the health and development of more than 100,000 children across the United States, following them from before birth until age 21 years. The EPA is participating in a collaborative effort with other government agencies to conduct the NCS and has an important role in environmental exposure assessment in the study. Initial efforts have been made on several tools to address Science Question no. 2, including a multi-pollutant Exposure Model for Individuals (EMI) initially focusing on asthma (Breen et al., 2008; http://birenheide.com/sra/ 2008AM/program/singlesession.php3?sessid = M2-F) and a community dietary questionnaire. Specific details on other models, measurements, and methods tools, and related potential case studies for the NCS and its Vanguard Centers are still being explored.

As a large-scale longitudinal epidemiological study that will collect a rich data set, the NCS presents a tremendous opportunity for assessing human exposures and risks and for evaluating estimates from tools such as the EMI and CFERST. Although the NCS focuses more on exposures at the individual level and CARE and other community programs focus more on community-level population estimates, many models and other tools being developed for both purposes (e.g., EMI and C-FERST) will overlap in terms of development, data use, and application. In both NCS epidemiology studies and CARE community applications (e.g., with C-FERST), human exposure estimates are needed for multiple pollutants and need to be considered cumulatively, across toxic substances and other stressors, and for a large number of health effects.

\section{Tools to Quantify Community Exposures and Impact of Reduction Actions}

The methods to be developed, applied, and provided under this project are grouped into several categories: specific guidance for community data collection and inexpensive screening-level methods for multiple chemicals. Based on the overview of methods presented in Medina-Vera et al. 2009, new inexpensive screening level methods for multiple chemicals will be developed and applied in selected community exposure case studies. For example, new cost-effective methodologies are needed to improve the human site-specific cumulative risk estimates associated with exposures to a variety of toxic element sources in soils and other environmental media. Other tools to address Science Question no. 3 include methods to link models and measurements for characterizing emissions, concentrations, and exposures for community risk assessments; new methods for continuous monitoring of multiple pollutants in communities (e.g., to identify "hot spots"); a cumulative community inhalation exposure model; and GIS tools for illustrating exposure and risk reduction scenarios.

\section{Discussion}

The EPA National Exposure Research Laboratory's Cumulative Communities Research Program was designed to provide exposure assessment tools (e.g., models, methods, data, GIS applications) to help address key needs in the areas of cumulative and community risk assessments. For example, the new C-FERST is a highly anticipated EPA tool that will provide maps and other information to depict relevant stressors and characterize cumulative exposures or risks across the country or at a community level (county or census tract). Visual maps can be very helpful for assisting communities in understanding and prioritizing their environmental issues for risk management decisions. Initial CFERST issues include benzene, radon, environmental tobacco smoke, ultraviolet radiation exposure due to stratospheric ozone depletion, diesel exhaust, cumulative childhood lead exposure, childhood asthma, and possibly particulate matter and ozone through collaboration with EPA Program Office and other experts. C-FERST will help with identifying communities at risk to multiple high priority chemicals, for predicting relative risk across regions and communities, and for assessing and ranking magnitude and contributions of multiple stressors. Such assessments could be used to help show the impact of Agency mitigation strategies 
on public health. Developed in close collaboration with CARE (the EPA's coordinated community program), and refined through future community case studies, C-FERST will bridge the gap between the emerging community-based cumulative risk science and actual use by the EPA's Regional Offices first, and then community groups at large. Much scientific research is needed to provide scientifically sound information (e.g., maps of concentrations, human exposures, and health risks) for a community report that is consistent across the many issues of interest.

Continued development and application of the new Exposure Model for Individuals (EMI) will provide estimates of exposure at the individual level in health studies such as the NCS for health effects such as asthma. Possible field studies to test low-cost measurement methods for community use in cumulative risk assessment would be helpful for a number of purposes: to conduct model evaluation at the personal and community population level; to serve as surrogates for personal exposure measurements; to provide modeled inputs for improved estimates of exposure at the personal and community level; to understand important sources, pathways, and factors for exposure; to provide information to validate field study sampling methods to correct for bias and imprecision in exposure-response relationships; to enhance understanding of contributions to observed biomarker data; to help assess community level impacts on exposures to relate community changes to expected exposures and health outcomes; and to provide guidance on optimal study designs to collect measurements needed in community field studies.

Research is also needed to improve the integration of exposure and spatial modeling tools with modifying factors (e.g., social science data) for characterizing how the complex interactions between environmental stressors and modifying factors impact human exposures and risks in the community setting. Upcoming EPA (NCER) grants for extramural research (http://es.epa.gov/ncer/rfa/\#CurrentStar; "Understanding the Role of Nonchemical Stressors and Developing Analytic Methods for Cumulative Risk Assessments") on the combined effects of toxic stressors and modifying factors will enable the analysis of disparate types of data involved in cumulative risk assessments; incorporation of this research into C-FERST and other tools developed under the CCRP is another important area for future research.

Future research could also include the following: applying source-to-health effect models and GIS tools for assessing and communicating the effectiveness of voluntary actions taken by community programs; developing and applying new models, methods, and data to identify, prioritize, and characterize cumulative exposures over time in support of the NCS Centers; developing and testing new methods for continuous monitoring of multiple pollutants in communities (e.g., to identify "hot spots"); applying available source apportionment and receptor models at the local scale in conjunction with local measurement data, to help identify important point sources of exposures within a given community; and applying the various tools to characterize cumulative exposures in collaborative studies (e.g., CARE; children's health research centers funded by the EPA and the National Institute of Environmental Health Sciences, http://www.niehs.nih.gov/research/ supported/centers/prevention/; ORD exposure and epidemiology studies; and EPA Program Office problem-driven scenarios).

The EPA research program described in this paper has potential for great impact as part of a cross-Agency effort (USEPA et al., 2007) to support community-based cumulative risk assessment. The research products developed through this program and related efforts outside of the Agency will advance the science for cumulative risk assessments and empower communities with information so that they can make informed, cost-effective decisions to improve public health.

\section{Acknowledgements}

We thank all the EPA scientists who contributed to the conceptual development of the NERL CCRP, especially Linda Sheldon, Roy Fortmann, Ross Highsmith, Hugh Tilson, Matthew Lakin, James Quackenboss, and Michael Callahan. We also thank the many NERL scientists on the Communities Research Team, especially those whose work has been particularly instrumental in the tools review, development of new tools, and case studies to date. These include Myriam Medina-Vera, Timothy Barzyk, Davyda Hammond, Michael Breen, Kathryn Conlon, and Teresa Chahine. In addition, we gratefully acknowledge our CARE and Regional collaborators, particularly Marybeth Smuts, Hank Topper, Deldi Reyes, Marva King, Carrie Knowlton, Matthew Lakin, and Debbie Bishop, the Community-Based Technical Support Forum, our NCER colleague Deborah Segal, and our Program Office collaborators, including Ted Palma and Cathy Fehrenbacher.

\section{Disclaimer}

The U.S. Environmental Protection Agency through its Office of Research and Development funded and managed the research described here. It has been subjected to Agency administrative review and approved for publication.

\section{References}

Barzyk T., Conlon K., Zartarian V., Lakin M., and Schultz B. Research Initiatives to Reduce Community Level Exposures: CARE and the Communities Project. Abstract 671. In: Abstracts of the 17th Annual Conference of the International Society of Exposure Analysis. Durham, NC, ISEA 2007.

Barzyk T., Conlon K., Chahine T., Hammond D., Zartarian V., and Schultz B. Tools Available to Communities for Conducting Cumulative Exposure and Risk Assessments, 2009, doi:JES.2009.25.

Breen M.S., Breen M., and Williams R.W. Air Pollution Exposure Model for Individuals (EMI) in Health Studies: Model Evaluation of Residential Air Exchange Rates. Society For Risk Analysis Annual Meeting 2008. 
Callahan M., and Sexton K. If cumulative risk assessment is the answer, what is the question? Environ Health Perspect 2007: 115(5): 799.

Davis R., Cook D., and Cohen L. A community resilience approach to reducing ethnic and racial disparities in health. Am J Public Health 2005: 95(12): 2168-2173.

DeFur P., Evans G., Cohen Hubal E., Kyle A., Morello-Frosch R., and Williams D. Vulnerability as a function of individual and group resources in cumulative risk assessment. Environ Health Perspect 2007: 115(5): 817.

Hammond D., Barzyk T., Conlon K., Zartarian V., and Schultz B. Application of GIS Mapping Tools to Prioritize Community Air Pollution Issues. Abstract \# 961. In: Abstracts of the ISEE/ISEA Joint Annual Conference, Pasadena, California. October 12-16, 2008.

Hammond D., Conlon K., Barzyk T., Chahine T., Zartarian V., and Schultz B. Application of national databases and mapping tools at the local level to two community case studies. Submitted for publication 2009 .

Hood E. Dwelling disparities: how poor housing leads to poor health. Environ Health Perspect 2005: 113(5): A310.

Lemay A., and Piotrowski J. The reconciliation model: a conceptual framework. Notre Dame Child Family Journal 2002: 7(3): 5-14.

Medina-Vera M., Van Emon J., Melnyk L., Bradham K., Harper S., and Morgan J. An overview of measurement tools available to communities for conducting exposure and cumulative risk assessments. J Expo Sci Environ Epidemiol, 2009, doi:JES.2009.23.

Menzie C., MacDonell M., and Mumtaz M. A phased approach for assessing combined effects from multiple stressors. Environ Health Perspect 2007: 115(5): 807.

Mokdad A., Marks J., Stroup D., and Gerberding J. Actual causes of death in the United States, 2000. JAMA 2004: 291: 1238-1245.

Mokdad A.H., et al. Correction: actual causes of death in the United States, 2000. JAMA 2005: 293: 293-294.

NACCHO (National Association of County and City Health Officials). Protocol for Assessing Community Excellence in Environmental Health: A Guidebook for Local Health Officials. National Association of County and City Health Officials CEHA Program. May 2000.

National Academy of Public Administration (NAPA). Evaluation of the "Community Action for Renewed Environment" Program, Phase I, 2008.

National Academy of Sciences (NAS). Science and Decisions: Advancing Risk Assessment. National Academies Press, 500 Fifth Street, NW, Washington, D.C., 2008.

National Children's Study. National Children's Study Research Plan. National Institute of Child Health and Human Development, 2007.

Sapolsky R. Sick of Poverty. Sci Am 2005: 293(6): 92-99.

USEPA. Unfinished Business: A Comparative Assessment of Environmental Problems. Washington, D.C. EPA, 1987 Feb. EPA \# 230287025a Available from: National Technical Information Service. Order No. PB88-127048 1987.

USEPA. Reducing Risk: Setting Priorities and Strategies for Environmental Protection. The report of the Science Advisory Board to William K. Reilly, Administrator. Washington, D.C., EPA, 1990 Sep. v, 27 p. (SAB-EC-90-
021PB91-155242) Available from: National Technical Information Service. Order No. PB91-155242.

USEPA. Guidebook to comparing risks and setting environmental priorities. EPA 230-B-93-003. Office of Policy, Planning and Evaluation USEPA, Washington DC, 1993.

USEPA. Framework for Cumulative Risk Assessment. EPA-630-P-02-001F. Risk Assessment Forum USEPA, Washington, DC, 2003.

USEPA. Community Action for a Renewed Environment Brochure, EPA-400-F-06003. USEPA, Washington DC, 2005.

USEPA. 2006-2011 EPA Strategic Plan: Charting Our Course. EPA-190-R-06001 USEPA, Washington DC, 2006a

USEPA. Human Health Research Program Multi-Year Plan (FY 2006-2013). Office of Research and Development. USEPA 2006b.

USEPA. National-Scale Air Toxics Assessment 1999, Office of Air and Radiation. USEPA 2006c.

USEPA, CDC, and ATSDR Memorandum of Understanding between the Centers for Disease Control and Prevention and the Agency for Toxic Substances and Disease Registry and the U.S. Environmental Protection Agency 2007.

USEPA. Concepts, Methods and Data Sources for Cumulative Health Risk Assessment of Multiple Chemicals, Exposures and Effects: A Resource Document. EPA/600/R-06/013F USEPA, Washington DC, 2007a.

USEPA. Proceedings of the U.S. EPA Workshop on Research Needs for Community-Based Risk Assessment, October 18-19, 2007 USEPA, Research Triangle Park, NC, 2007b.

USEPA. EPA's 2008 Report on the Environment (Final Report). EPA/600/R-07/ 045F Washington DC, 2008a.

USEPA. The CARE Roadmap: 10-Step Plan to Improve Community Environment and Health, EPA 400-K-08-002. USEPA 2008b.

USEPA. The Community CARE Resource Guide. Washington, DC, USEPA, 2008c. www.epa.gov/osp/care/library/CARE_Resource Guide.pdf

Zartarian V., Schultz B., Quackenboss J., and Sheldon L. EPA/ORD/NERL Research Program on Exposure Tools to Advance the Science and Understanding of Cumulative Risk to Communities and Individuals. Abstract 613. In: Abstracts of the 17th annual conference of the International Society of Exposure Analysis. Durham, NC, ISEA 2007.

Zartarian V., and Schultz B. EPA's Community-Friendly Exposure and Risk Screening Tool. Abstract no. 1018. In: Abstracts of the ISEE/ISEA Joint Annual Conference, Pasadena, California. October 12-16, 2008.

This work is licensed under the Creative Commons Attribution-NonCommercial-No Derivative Works

3.0 License. To view a copy of this license, visit http:// creativecommons.org/licenses/by-nc-nd/3.0/ 